ReVista de Matemática: TeOría y APliCACiones 2016 23(1) : 255-276

CIMPA - UCR ISSN: 1409-2433 (PRINT), 2215-3373 (ONLINE)

\title{
EL MODELO LOGÍSTICO MIXTO PARA PREDECIR CRISIS FINANCIERA EN EMPRESAS ARGENTINAS Y CHILENAS
}

\section{MIXED LOGISTIC MODEL FOR PREDICTING FINANCIAL CRISIS IN ARGENTINEAN AND CHILEAN ENTERPRISES}

\author{
NORMA PATRICIA CARO*
}

Received: 28 Mar 2014; Revised: 28 Aug 2015;

Accepted: 1 Oct 2015

*Facultad de Ciencias Económicas, Universidad Nacional de Córdoba. Córdoba, Argentina. E-Mail: pacaro@eco.unc.edu.ar 


\title{
Resumen
}

Desde la década de 1960, las empresas tienen como objetivo evaluar los resultados futuros del gerenciamiento empresarial para predecir, a mediano plazo, procesos de gestación e instalación de estados de vulnerabilidad financiera. La información contenida en los estados financieros de las empresas y la posibilidad de analizar la evolución en el tiempo de los ratios contables permiten construir modelos de predicción de riesgo de crisis financiera. En este trabajo, se construye un modelo de predicción de riesgo con base en la información contenida en los Estados Contables de las empresas con oferta pública en las Bolsas de Santiago de Chile y de Valores de Buenos Aires (Argentina) para la década del 2000.

La crisis financiera se caracteriza por la incapacidad de cumplir con las obligaciones de pago, la obtención de magnitudes excesivas de pérdidas y por situaciones extremas como la quiebra y posterior liquidación de la empresa. Hasta hace un poco más de un año, la mayoría de los trabajos desarrollados para cuantificar la incidencia de ratios financieros en la crisis empresarial, aplicaron métodos de corte transversal, por lo que la construcción de modelos para datos longitudinales resulta pertinente, en tanto incorporan la dimensión temporal en el estudio. En particular, se ha demostrado que el modelo logístico mixto, que tiene en cuenta la heterogeneidad no observada supera ampliamente el desempeño del modelo logístico estándar.

Tanto en Argentina como en Chile se han aplicado, recientemente, modelos mixtos con coeficientes aleatorios para predecir estados de vulnerabilidad financiera.

Los resultados obtenidos indican que, en las empresas chilenas, el ratio del capital de trabajo explica la mayor proporción de la heterogeneidad inducida por la correlación que presentan los datos, lo que justifica su inclusión como coeficiente aleatorio, mientras que en el mercado argentino lo es el índice de rentabilidad. Además, como efectos fijos, los indicadores con mayor capacidad predictiva de la crisis financiera son los índices de rentabilidad, rotación y endeudamiento.

Se concluye que, los ratios significativos poseen poder discriminatorio y su comportamiento muestra que son indicadores para la predicción de crisis.

Palabras clave: modelo lineal generalizado mixto; coeficientes aleatorios; ratios financieros; crisis financiera.

\begin{abstract}
Since the 1960s, companies aim to evaluate future performance of the business management to predict the medium term, processes of gestation and installation of statements of financial vulnerability.
\end{abstract}


The information contained in the financial statements of companies and the ability to analyze the evolution in time of financial ratios allows building models predicting risk of financial crisis.

This paper presents a risk prediction model based on the information contained in the financial statements of companies with public offering on the Santiago Stock Exchange and the Stock Exchange of Buenos Aires (Argentina) in the 2000s.

The financial crisis is characterized by an inability to meet payment obligations, obtaining excessive quantities of waste and in extreme situations like bankruptcy and subsequent liquidation of the company.

Until a little over a year ago, most of the work done to quantify the impact of financial ratios in business crisis apply cross-sectional models, but the construction of models for panel data (longitudinal studies) is relevant given that incorporate the temporal dimension in the study. In particular, it has been demonstrated that the mixed logistic model considered unobserved heterogeneity exceeds the performance of standard logistic model.

Both Argentina and Chile have recently applied mixed models with random coefficients to predict statements of financial vulnerability.

The results indicate that in Chilean companies, the ratio of working capital accounts for the largest proportion of heterogeneity induced correlation present data, which justifies its inclusion as random coefficient, while in the Argentine market it is profitability rate. In addition, as fixed effects, indicators best predictor of the financial crisis they are profitability ratios, rotation and debt.

It is concluded that significant ratios have discriminatory power and their behavior shows that are indicators for predicting crises.

Keywords: generalized linear mixed model; random coefficients; financial ratios; financial crisis.

Mathematics Subject Classification: 62P20, 91B82, 62M20.

\section{Introducción}

Desde mediados de siglo pasado las empresas han evaluado los resultados del gerenciamiento empresarial para predecir, a mediano plazo, procesos de gestación e instalación de estados de vulnerabilidad financiera.

La información contenida en los Estados Contables de las empresas y la posibilidad de analizar la evolución en el tiempo de los ratios contables permiten construir modelos de predicción de riesgo de crisis financiera.

En este trabajo se construye un modelo de predicción de riesgo con base en la información contenida en los Estados Contables de las empresas con oferta 
pública en la Bolsa de Santiago de Chile para la década del 20001, a fin de detectar si las empresas poseen un estado de crisis financiera y analizar los ratios contables que inciden en la probabilidad de la misma.

Se define la crisis financiera como la incapacidad de cumplir con las obligaciones de pago, la obtención de magnitudes excesivas de pérdidas y la declaración de quiebra de la empresa.

Así, surgieron numerosos trabajos orientados a la elaboración de modelos que permiten anticiparse a procesos de insolvencia utilizando como base la información contenida en los balances, con la que se construyen ratios económicos/financieros.

Con respecto a la metodología, existen dos etapas que reflejan la evolución de la misma. Una etapa descriptiva, alrededor de los años treinta, que consistió en el cálculo de los ratios y su análisis en forma individual en los distintos periodos contables; y con posterioridad, la etapa predictiva llevada a cabo por Beaver (1966) [5] y Altman (1968) [1], en los años sesenta, donde se empezó a relacionar el significado de cada uno de esos ratios para explicar la quiebra de las empresas a través de estos indicadores y por lo tanto su posibilidad de predicción. En términos generales estos modelos son utilizados con diferentes propósitos, en entidades financieras para el análisis de riesgo crediticio de las carteras de créditos, en los entes calificadores de deuda, en las compañías de seguro, en el análisis de precios de bonos, entre otros. La quiebra o default no solamente interesa a los agentes que están relacionados en forma directa con ellos, como inversores, clientes o proveedores, sino también en forma indirecta con la sociedad en general ante las consecuencias negativas que provoca la quiebra de una empresa.

En este trabajo se busca obtener indicadores determinantes de la crisis financiera de las empresas de Chile a través de la construcción de un modelo de predicción, para luego ver las posibles diferencias o similitudes de estos factores, con los que se determinaron en las empresas argentinas (Caro, 2013) [7].

Partiendo de la hipótesis de que los indicadores financieros son los adecuados para caracterizar el comportamiento de las empresas y poder anticiparse a situaciones de vulnerabilidad financiera, surge la pregunta que se abordará en este trabajo de investigación, ¿son los ratios contables, los indicadores válidos para predecir la crisis financiera?

Para responder a esta pregunta, es necesario construir un modelo de predicción, propio para la economía chilena, lo que se realiza a través de un modelo

\footnotetext{
${ }^{1}$ Primeros avances presentados en las XXXIV Jornadas Universitarias de Contabilidad, Santa Fe, Argentina, bajo el titulo: "Importancia de los ratios contables en la evaluación de riesgo de crisis financiera en empresas chilenas en la década del 2000. Su comparación con las empresas argentinas".
}

Rev.Mate.Teor.Aplic. (ISSN print: 1409-2433; online: 2215-3373) Vol. 23(1): 255-276, January 2016 
logístico mixto, siendo la variable respuesta, el estado de la empresa (con problemas financieros o sin ellos) y las variables independientes, los ratios que se utilizan en estos modelos en otras economías, según lo expresado en la literatura (Jones y Hensher, 2004) [11].

Los ratios que resultan significativos poseen poder discriminatorio. De esta manera se contribuye con el análisis de los estados contables como herramienta de predicción de crisis financiera.

La estructura del este artículo posee una introducción, una sección que comenta sobre los modelos estadísticos de predicción de crisis en economías desarrolladas y emergentes; una tercera parte que comprende los principales aspectos del método, las variables que se utilizan y los modelos aplicados; una cuarta sección, con los resultados obtenidos y por último las conclusiones a las que se arriba.

\section{Antecedentes: Modelos estadísticos de predicción de crisis}

Las primeras investigaciones datan de comienzos de la década de 1930, período caracterizado como la etapa descriptiva, donde las investigaciones sobre la situación financiera de las empresas se centraron en la tendencia de los ratios. Se utilizaron métodos univariados analizando cada ratio, en forma individual, y su comportamiento en el grupo de las empresas con problema financieros, comparativamente al grupo de las empresas sanas.

A partir de la década de 1960 se desarrolla la etapa predictiva donde se analiza la significatividad de cada índice, es decir la capacidad que tiene para predecir la quiebra de una empresa. Se construyeron modelos de predicción de riesgo tanto en economías desarrolladas como en países emergentes.

Beaver (1968) [6] y Altman (1968)[1] comenzaron con el desarrollo de estos modelos en economías desarrolladas, los que se fueron adaptando a economías emergentes. Altman (1968) [1] comienza a aplicar herramientas estadísticas multivariadas, como el análisis discriminante para lograr el objetivo de la clasificación de las empresas según su riesgo de quiebra. Este modelo fue utilizado en diferentes países, en estudios de análisis de riesgo financiero, hasta llegar a ser aplicado en empresas de economías emergentes.

Jones y Hensher (2004) [11], en Australia, trabajaron con dos muestras, una de estimación y otra de validación, donde la variable respuesta es definida en tres estados de situación financiera: no fallidas, insolventes y quebradas o en proceso judicial. El periodo de análisis fue entre 1996 a 2000 y la información contable que se utilizó fue por un periodo de 5 años. Aplicaron modelos mixtos y sus 
conclusiones muestran, que los indicadores explicativos de situaciones de crisis, son aquellos que recogen información de efectivo o liquidez, flujo de fondos provenientes de las operaciones, capital de trabajo, rentabilidad, volumen del negocio, endeudamiento y capacidad de pago de los servicios de la deuda. Este trabajo fue el que motivó la aplicación de estos modelos en economías emergentes, ya que se prueba la mejor performance de los mismos con respecto a los modelos de análisis discriminante y logístico.

Dadas las diferentes crisis que se sucedieron en las economías emergentes, se empiezan a aplicar estos modelos de predicción, a fines de la década del 70. En una primera instancia se utilizó el modelo tradicional de Altman, "Z-Score", no obstante al ser diseñado para empresas que se desarrollan en ambientes completamente diferentes, fue necesario adaptarlo a estas nuevas economías. Fue así que surgieron nuevos trabajos de investigación (Altman, Baida y Rivero, Díaz, 1979[3]; Swanson y Tybout, 1988 [17]; Pascale, 1988 [14]; Romani Chocce y Aroca Gonzales, 2002 [15]; Sandín y Porporato, 2007 [16]; Zurita, 2008 [18] y Mongruta Montalván, Alberti Delgado, Fuenzalida O’Shee y Akamine Yamashiro, 2011 [13]) con el objetivo de estudiar el comportamiento de los indicadores contables para predecir el riesgo de insolvencia.

Respecto a la etapa predictiva, en un comienzo, se aplicaron modelos de clasificación supervisada para datos de corte transversal (análisis discriminante lineal, cuadrático, regresión logística, árboles de clasificación, entre otros), a un solo periodo, en el que se manifestaba la crisis, o bien un año antes, o dos, o más. Luego se compararon los resultados obtenidos en cada año y se concluyó, que mientras más cerca se aproximaba al momento de la crisis, el modelo clasificaba mejor, con mayor tasa de clasificación correcta.

Luego, en la década del 2000, se empezaron a aplicar modelos que tienen en cuenta la historia de la empresa, es decir la información contable de varios años anteriores al momento de la manifestación de la crisis, lo que obligó a trabajar con observaciones no independientes. Es decir, considerando un año, dos o tres antes de la manifestación de crisis, los modelos para datos longitudinales como los mixtos y los de riesgo resultan más apropiados para explicar y predecir la crisis financiera.

Muchas de estas investigaciones se encuentran mencionadas en Caro (2014) [9].

\section{Método}

Se trabajó con las empresas que cotizan en Bolsa y publican sus Estados Contables en la "Superintendencia de Valores y Seguros" de Santiago de Chile. Se 
excluyó del trabajo, las entidades financieras, compañías de seguro y las administradoras de fondos y pensiones, por estar sujetas a un sistema de regulación particular y específico para cada actividad (Altman, 1968) [1].

Del total de empresas que cotizan en la Bolsa de Chile, para el periodo 2000 - 2010, se seleccionaron, en primer lugar, las empresas en crisis a través del análisis de la información publicada en la Bolsa y de sus estados de resultados y en segundo lugar las empresas sanas.

Con respecto a las primeras, se seleccionan 22 empresas (Tabla 11), de las cuales se tomaron los periodos anteriores a la manifestación de la crisis, acotando un máximo de 6 estados contables. De las empresas sanas se seleccionó una muestra de 89 empresas que no manifiestan problemas financieros y de ellas una cantidad de periodos similares a los considerados para las empresas en crisis.

Las empresas no poseen la misma cantidad de balances, tienen un mínimo de 2 y un máximo de 6 , depende de la cantidad de años disponibles en el período 2003 al 2010 y del momento en que la empresa manifiesta su estado de crisis.

Tabla 1: Empresas de la muestra.

\begin{tabular}{c|ccc}
\hline \hline & $\begin{array}{c}\text { Sin problemas } \\
\text { financieros (sanas) }\end{array}$ & $\begin{array}{c}\text { Con problemas } \\
\text { financieros (en crisis) }\end{array}$ & Total \\
\hline $\begin{array}{c}\text { Empresas } \\
\text { Observaciones } \\
\text { (balances) }\end{array}$ & 89 & 22 & 111 \\
\hline \hline
\end{tabular}

\subsection{Las variables del modelo}

La variable respuesta es de característica dicotómica. Se considera que una empresa está en crisis cuando se manifiestan problemas de cesación de pagos, como la solicitud de concurso preventivo, quiebra, pérdidas anuales consecutivas que afecten el patrimonio de la empresa.

El "Manual de Derechos y obligaciones de emisores de la Bolsa de Comercio de Santiago"2 en su artículo 10 (Título VI De las Sanciones) indica que "El Directorio podrá suspender la transacción y cotización oficial de alguno o de todos los valores emitidos, en caso de insolvencia, quiebra, cesación de pagos del emisor, realización de actividades prohibidas por parte de éste u otra causal grave que así lo exija en protección de los inversionistas."

Si bien en un grado menor al concurso preventivo o a la quiebra, no cabe duda que la obtención de pérdidas reiteradas o la cesación de pagos constituye

\footnotetext{
${ }^{2}$ Aprobado y con fecha de aplicación el 01 de marzo de 1988. Actualizado el 23 de septiembre de 2010.

Rev.Mate.Teor.Aplic. (ISSN print: 1409-2433; online: 2215-3373) Vol. 23(1): 255-276, January 2016
} 
un signo ostensible de vulnerabilidad financiera, razón por la cual se califica como empresa en crisis aquella que, durante el período de análisis manifestó alguna de estas situaciones.

Las variables independientes consideradas son:

\section{- Ratios Financieros}

Los ratios financieros de las empresas calculados con base en la información suministrada por los estados contables son las variables independientes del modelo de predicción. Uno de los problemas en las distintas investigaciones fue determinar el número adecuado de ratios y seleccionar aquellos que poseen un mayor poder de predicción. Existe una significativa cantidad de ratios, incluso muchos de ellos son equivalentes, ya que conceptualmente miden lo mismo.

En este trabajo se utilizan los mismos ratios de Jones y Hensher (2004) [11] y Altman (1968) [1] con algunas adaptaciones, a fin de poder comparar la aplicación del modelo en las empresas argentinas respecto a las empresas chilenas y que se detallan en la Tabla2.

Es importante aclarar, que no es posible decir que estos ratios tienen el mismo comportamiento en todos los mercados. En Argentina, los ratios que miden rentabilidad (GE_AT) y flujo de fondos operativos (FF_AT) resultan ser las más importantes a la hora de predecir el estado de la empresa, en el mismo período. (Caro, 2013) [7].

\section{- Características de las empresas}

Otras variables a incluir son las características de las empresas, el tamaño y el sector de la economía, en este caso se considera el tipo de actividad, por no contar con demasiadas empresas por sector (Tabla 4). En cuanto al tipo de actividad se las clasifica, por un lado, en actividades primarias y actividades relacionadas a los servicios públicos (eléctricas, gas, telefónicas), por otro lado, industrias y por último en el sector de servicios en general (Tabla 3).

El tamaño de la empresa fue incorporado por Altman (1977) [2], medido a través del logaritmo natural del activo total. 
Tabla 2: Ratios financieros utilizados.

\begin{tabular}{|c|c|c|}
\hline Ratio & Fórmula & Concepto \\
\hline Flujo de fondos & $\begin{array}{l}F F_{-} A T=\frac{F F O}{A T} \times 100 \\
F F O: \text { Flujos de fondo ge- } \\
\text { nerados por las operaciones. } \\
A T: \text { Activo Total }\end{array}$ & $\begin{array}{l}\text { Es la relación entre el flujo de fondos gene- } \\
\text { rados por las operaciones respecto del total } \\
\text { de activo, en porcentaje. Se espera que el } \\
\text { ratio sea negativo en las empresas en crisis. }\end{array}$ \\
\hline Liquidez & $\begin{array}{l}\quad E \_A T=\frac{E}{A T} \times 100 \\
E: \text { disponibilidades (efec- } \\
\text { tivo e inversiones que se } \\
\text { convierten en efectivo den- } \\
\text { tro de las } 24 \mathrm{hs).}\end{array}$ & $\begin{array}{l}\text { Mide la relación entre los recursos de } \\
\text { mayor disponibilidad respecto al activo to- } \\
\text { tal, en porcentaje. Se espera que el ratio se } \\
\text { positivo pero pequeño en las empresas en } \\
\text { crisis. }\end{array}$ \\
\hline $\begin{array}{l}\text { Rotación } \\
\text { sobre } A T\end{array}$ & $\begin{array}{l}\quad V_{-} A T=\frac{V}{A T} \times 100 \\
V: \text { total de ventas. }\end{array}$ & $\begin{array}{l}\text { Expresa el uso eficiente de los recursos } \\
\text { de la empresa en generar ventas, en por- } \\
\text { centaje. Se espera que el ratio sea positi- } \\
\text { vo, pero pequeño en las empresas en crisis. } \\
\text { Se ha,observado en algún caso que el nivel } \\
\text { de ventas ha sido nulo en las empresas con } \\
\text { problemas. }\end{array}$ \\
\hline Endeudamiento & $\begin{array}{l}\quad D \_P N=\frac{D}{P N} \times 100 \\
D: \text { total de deudas } P N: \text { Pat- } \\
\text { rimonio Neto }\end{array}$ & $\begin{array}{l}\text { Mide en cuanto se encuentra endeudada la } \\
\text { empresa por cada peso aportado por los } \\
\text { propietarios, en porcentaje. Se espera que } \\
\text { este ratio sea elevado en las empresas en } \\
\text { crisis. }\end{array}$ \\
\hline $\begin{array}{l}\text { Capital de } \\
\text { trabajo }\end{array}$ & $\begin{array}{l}\quad C T \_A T=\frac{C T}{A T} \times 100 \\
C T: \text { Capital de Trabajo (Ac- } \\
\text { tivo corriente menos pasivo } \\
\text { corriente) }\end{array}$ & $\begin{array}{l}\text { Mide la relación entre el capital de corto } \\
\text { plazo con el total de Activo de la empresa, } \\
\text { en porcentaje. }\end{array}$ \\
\hline $\begin{array}{l}\text { Rentabilidad } \\
\text { económica }\end{array}$ & $\begin{array}{l}\quad E \_A T=\frac{U a i I}{A T} \times 100 \\
\text { UaiI: utilidad antes de in- } \\
\text { tereses e impuestos }\end{array}$ & $\begin{array}{l}\text { Mide la rentabilidad de cada peso de activo } \\
\text { total, en porcentaje. }\end{array}$ \\
\hline
\end{tabular}


Tabla 3: Cantidad de empresas por sectores.

\begin{tabular}{c|ccc}
\hline \hline Sector & $\begin{array}{c}\text { Empresas } \\
\text { sanas }\end{array}$ & $\begin{array}{c}\text { Empresas } \\
\text { en crisis }\end{array}$ & Total \\
\hline \hline Act. primarias y & 33 & 5 & 38 \\
Servicios públicos & 33 & 10 & 43 \\
Industrial & 33 & 7 & 30 \\
Servicios en general & 23 & 22 & 111 \\
Total & 89 &
\end{tabular}

Tabla 4: Características de la empresa.

\begin{tabular}{|c|c|c|}
\hline Variable & Descripción & Concepto \\
\hline Sector2 & $\begin{array}{l}\text { Actividades primarias y } \\
\text { Servicios públicos } \\
\text { Industria }\end{array}$ & $\begin{array}{l}\text { Variable Dummy que compara las } \\
\text { empresas de cada sector con las del } \\
\text { sector de actividades primarias y } \\
\text { relacionadas a servicios públicos }\end{array}$ \\
\hline Sector3 & Servicios en general & \\
\hline LNAT & $\begin{array}{l}\text { Tamaño: } \\
\qquad L N A T=\ln A T\end{array}$ & $\begin{array}{l}\text { Mide el tamaño de las empresas a } \\
\text { través del Activo Total (AT) en lo- } \\
\text { garitmo. }\end{array}$ \\
\hline
\end{tabular}

\subsection{Modelos mixtos}

Se aplica la metodología de los modelos mixtos para predecir la crisis financiera en las empresas del mercado chileno a fin de comparar con los modelos aplicados en las empresas argentinas.

Para evaluar el riesgo de crisis se desarrollan modelos adecuados que utilizan la información proveniente de los estados contables. Por otro lado, los modelos estadísticos captan en forma real el comportamiento de las empresas, el tipo de actividad y otros indicadores que afectan el estado de las mismas. La complementación de ambos aspectos permite minimizar la tasa de error de clasificar una empresa como sana cuando en realidad tiene dificultades financieras.

En este trabajo se utiliza un modelo donde la variable respuesta es la variable binaria que indica el grupo al que pertenece la empresa (en crisis $=1 /$ sanas $=$ 0 ), y las variables predictoras que son los indicadores económico-financieros y características de la empresa. Los problemas con respuesta dicotómica son 
modelados usualmente a través de regresión logística, donde la probabilidad de que la variable respuesta $\left(y_{i}\right)$ asuma el valor 1 , suponiendo sólo una variable independiente o regresoras $\left(x_{i}\right)$ en el predictor lineal, es:

$$
\operatorname{Pr}\left(Y_{i}=1 / X_{i}\right)=h\left(\beta_{1}+\beta_{2} x_{i}\right)=h\left(z_{i}\right) \quad i=(1,2, \ldots, n),
$$

siendo la transformación logística:

$$
\operatorname{logit}\left(\operatorname{Pr}\left(Y_{i}=1 / X_{i}\right)\right)=\ln \left(\frac{\operatorname{Pr}\left(y_{i}=1 / x_{i}\right)}{1-\operatorname{Pr}\left(y_{i}=1 / x_{i}\right)}\right)=z_{i} .
$$

El modelo logístico supone que las respuestas son independientes dadas las variables regresoras, por lo que resulta apropiado cuando los datos no tienen ningún tipo de agrupamiento. Este requisito no se cumple en este trabajo, ya que la estructura de los datos introduce dependencia en las respuestas múltiples dentro de cada unidad, aún condicionando sobre dichas variables.

El modelo general con un coeficiente aleatorio en el predictor lineal, presenta la siguiente estructura:

$$
P\left(Y_{i}=1 / X_{i}\right)=\beta_{0}+\left(\beta_{1}+b_{1 j}\right) X_{1 i j}+\beta_{2} X_{2 i j}+\ldots+\beta_{k} X_{k i j}
$$

donde, el efecto aleatorio posee distribución normal.

$$
b_{j} \sim N\left(0, \sigma^{2}\right)
$$

siendo

$i$ : observación, en este caso periodo anual,

$j$ : empresa,

$k$ : variable regresora. donde

$\beta_{k}$ : coeficientes de cada una de las variables independientes,

$b_{j}$ : efecto aleatorio de cada empresa,

$x_{k i j}: k$ variables independientes del $i$-ésimo periodo de la $j$-ésima empresa.

Los efectos aleatorios son predichos y en función de dichas predicciones se evalúa el modelo, a fin de determinar si las empresas son correctamente clasificadas en el estado correspondiente.

En función de lo expresado, se han aplicado dos modelos, uno con ratios financieros y variables que caracterizan a la empresa a los fines de compararlo con el modelo aplicado a las empresas argentinas y un segundo modelo solo con ratios con el objeto de determinar el poder predictivo de los indicadores financieros. 


\section{Resultados}

Realizado un análisis descriptivo de cada grupo de empresas, se obtienen los siguientes resultados (Tabla5):

El índice de rentabilidad promedio de las empresas en crisis es negativo, del $10,11 \%$ debido al nivel de pérdidas obtenidas en el período, contra un $6,41 \%$ positivo de las empresas sanas. La mediana asciende a $-1,36 \%$, lo que implica que existen valores extremos. Es significativa la dispersión en el grupo de las empresas en crisis en comparación a las sanas.

El índice del flujo de fondos operativos promedio en las empresas en crisis es negativo del $-9,29 \%$, lo que significa que el flujo de fondos generado por las operaciones no alcanza a cubrir las erogaciones de las mismas contra un promedio de 9,36\% en las empresas sanas. En las empresas en crisis esta variable presenta una altísima variabilidad relativa respecto al grupo de empresas sanas y valores extremos negativos, por lo que la mediana resulta menor que la media.

El nivel de efectivo promedio es similar en ambos grupos de empresas, incluso se llega a la misma conclusión si observamos su variabilidad.

El volumen promedio de ventas respecto al total de activo es menor $(33,04 \%)$ en las empresas en crisis en relación con las empresas sanas $(57,64 \%)$, es decir que el volumen de negocios de las empresas sanas es 1,74 veces mayor al resultante de las empresas con problemas financieros.

El endeudamiento promedio es 5,30 veces superior en las empresas en crisis que en las empresas sanas. Es significativa la variabilidad en el primer grupo con valores máximos extremos, situación que se refleja en la mediana de 64,87\% contra un promedio de $371,66 \%$. Esta variable refleja una notable diferencia entre ambos grupos, dónde las empresas en crisis se caracterizan por el fuerte endeudamiento al que han tenido que recurrir para mantener sus niveles operativos.

El ratio del capital de trabajo promedio, al igual que el índice de liquidez, es similar en ambos grupos, aunque con mayor dispersión en las empresas con problemas. 
Tabla 5: Estadística descriptiva de los Ratios - Chile.

\begin{tabular}{|c|c|c|}
\hline \multirow[t]{2}{*}{ Medidas } & \multicolumn{2}{|c|}{ Estado } \\
\hline & Sanas & En Crisis \\
\hline Observaciones & 522 & 111 \\
\hline \multicolumn{3}{|c|}{ Indice de Rentabilidad (GE_AT) } \\
\hline Media & 6,41 & $-10,107$ \\
\hline Mediana & 5,946 & $-1,359$ \\
\hline Desviación estándar & 10,739 & 31,307 \\
\hline Coeficiente de variación & 1,675 & 3,098 \\
\hline Valor Mínimo & $-147,35$ & $-271,554$ \\
\hline Valor Máximo & 85,997 & 21,801 \\
\hline \multicolumn{3}{|c|}{ Indice de Flujo de Fondos (FF_AT) } \\
\hline Media & 9,357 & $-9,286$ \\
\hline Mediana & 8,274 & $-0,723$ \\
\hline Desviación estándar & 9,823 & 36,918 \\
\hline Coeficiente de variación & 1,050 & 3,976 \\
\hline Valor Mínimo & $-29,899$ & $-266,7$ \\
\hline Valor Máximo & 126,008 & 83,329 \\
\hline \multicolumn{3}{|c|}{ Indice de Liquidez (E_AT) } \\
\hline Media & 1,074 & 1,013 \\
\hline Mediana & 0,428 & 0,362 \\
\hline Desviación estándar & 2,722 & 2,609 \\
\hline Coeficiente de variación & 2,534 & 2,576 \\
\hline Valor Mínimo & 0 & 0 \\
\hline Valor Máximo & 34,076 & 25,734 \\
\hline \multicolumn{3}{|c|}{ Rotación sobre Activo Total (V_AT) } \\
\hline Media & 57,639 & 33,039 \\
\hline Mediana & 50,707 & 23,400 \\
\hline Desviación estándar & 53,891 & 38,125 \\
\hline Coeficiente de variación & 0,935 & 1,154 \\
\hline Valor Mínimo & 0 & 0 \\
\hline Valor Máximo & 387,255 & 154,954 \\
\hline \multicolumn{3}{|c|}{ Indice de Endeudamiento (D_PN) } \\
\hline Media & 70,364 & 371,659 \\
\hline Mediana & 56,969 & 64,871 \\
\hline Desviación estándar & 58,43 & 2018,65 \\
\hline Coeficiente de variación & 0,830 & 5,431 \\
\hline Valor Mínimo & 0,056 & 0,039 \\
\hline Valor Máximo & 419,944 & 21167,5 \\
\hline \multicolumn{3}{|c|}{ Capital de Trabajo (CT_AT) } \\
\hline Media & 11,983 & 11,68 \\
\hline Mediana & 7,842 & 4,457 \\
\hline Desviación estándar & 14,388 & 36,634 \\
\hline Coeficiente de variación & 1,201 & 3,135 \\
\hline Valor Mínimo & $-18,773$ & $-90,853$ \\
\hline Valor Máximo & 93,089 & 99,95 \\
\hline
\end{tabular}

Rev.Mate.Teor.Aplic. (ISSN print: 1409-2433; online: 2215-3373) Vol. 23(1): 255-276, January 2016 
A continuación se presentan los modelos aplicados en las empresas chilenas. Por un lado, el modelo con todas las variables independientes explicadas en la sección anterior (ratios y características de la empresas). En segundo lugar, se presenta el modelo construido con empresas Argentinas (Caro, 2013) [7] y por último un modelo aplicado a las empresas chilenas, sólo con ratios, a fines de comparar con las empresas argentinas.

\subsection{Modelo con ratios, tamaño y sector en empresas chilenas}

El modelo aplicado es:

$$
\begin{aligned}
P\left(Y_{i}=1 / \mathbf{X}_{i j}\right)= & \beta_{0}+\left(\beta_{1}+b_{1 j}\right) C T_{-} A T_{i j}+\beta_{2} G E_{-} A T_{i j}+\beta_{3} F F_{-} A T_{i j} \\
& +\beta_{4} E_{-} A T_{i j}+\beta_{5} V_{-} A T_{i j}+\beta_{6} D_{-} P N_{i j}+\beta_{7} L N A T_{i j} \\
& +\beta_{8} S e c 2_{i j}+\beta_{9} S e c 3_{i j}
\end{aligned}
$$

donde $\mathbf{X}_{i j}$ representa el vector de covariables descriptas en las tablas $2 \mathrm{y} 4$

La estimación del modelo se obtuvo con el método de máxima verosimilitud utilizando la rutina NLMIXED del software SAS 3 , siendo las variables independientes los ratios contables, el sector y el tamaño de las empresas.

Los resultados (Tabla 6) muestran que el ratio que mide capital de trabajo $\left(C T \_A T\right)$ resulta adecuado para explicar la mayor proporción de la heterogeneidad inducida por la correlación que presentan los datos, lo que justifica su inclusión como coeficiente aleatorio.

La elección de este modelo se hizo construyendo pruebas de razón de verosimilitud $(L R T)$ y contrastando secuencialmente contra modelos sin efectos aleatorios.

La inclusión de este coeficiente aleatorio permitió identificar aquellos indicadores con mayor capacidad predictiva de la crisis financiera de las empresas. Se advierte que los ratios de rentabilidad $\left(G E \_A T\right)$, nivel de ventas respecto del activo total $\left(V_{-} A T\right)$, endeudamiento $\left(D_{-} P N\right)$, capital de trabajo $\left(C T \_A T\right)$, tamaño $(L N A T)$ y los sectores industriales $(S e c 2)$ son significativos con respecto al sector primario más el relacionado a servicios públicos que fue considerado el sector de referencia $(\alpha=0,05)$. Como se observa en la Tabla 6 el signo negativo de los coeficientes de los ratios significativos indica que un aumento en los mismos disminuye la chance de crisis, excepto el caso del endeudamiento, cuyo signo positivo está indicando que un aumento en el ratio, aumenta la chance de crisis.

\footnotetext{
${ }^{3}$ Statistical Analysis System provisto por SAS Institute Inc., es un lenguaje de programación que opera sobre base de datos.
} 
Tabla 6: Estimación de los parámetros del modelo logístico mixto - Chile.

\begin{tabular}{c|cccc}
\hline \hline $\begin{array}{c}\text { Variables } \\
\text { independientes }\end{array}$ & Coeficiente & $\begin{array}{c}\text { Error } \\
\text { Estándar }\end{array}$ & $\mathrm{P}>\mathrm{z}$ & $\begin{array}{c}\text { Odd ratios de } \\
\text { variables } \\
\text { significativas }\end{array}$ \\
\hline LNAT & $-0,6864$ & 0,2156 & $\mathbf{0 , 0 0 1 9}$ & 0,503 \\
GE_AT & $-0,6281$ & 0,151 & $\mathbf{0 , 0 0 0 1}$ & 0,534 \\
FF_AT & 0,0015 & 0,0377 & 0,9694 & \\
E_AT & 0,2519 & 0,3122 & 0,4214 & \\
V_AT & $-0,0496$ & 0,0178 & $\mathbf{0 , 0 0 6 2}$ & 0,952 \\
D_PN & 0,0164 & 0,0057 & $\mathbf{0 , 0 0 4 8}$ & 1,017 \\
CT_AT & $-0,2721$ & 0,1239 & $\mathbf{0 , 0 3 0 2}$ & 0,762 \\
Sec2 & 3,5249 & 1,0187 & $\mathbf{0 , 0 0 0 8}$ & 33,95 \\
Sec3 & 1,7767 & 1,0698 & 0,0996 & \\
Constante & 8,8721 & 4,0915 & 0,0323 & \\
\hline Efecto & & Error & LR test vs \\
Aleatorio & Varianza & Estándar & logistic regression \\
CT_AT & 0,2634 & 0,1306 & \multicolumn{2}{c}{ Pr (chibar2) } \\
\hline \hline
\end{tabular}

Efectuando un ordenamiento de los ratios según su capacidad discriminatoria, en primer lugar se posiciona el índice que mide Tamaño $(L N A T)$, el cual indica que por cada incremento porcentual en ese indicador, la chance de entrar en estado de crisis disminuye en un 49,7\%. En segundo lugar el índice de Rentabilidad ( $\left.G E \_A T\right)$, cuyo odd ratid 4 de 0,534 muestra que un incremento unitario en dicho índice disminuirá esa chance en aproximadamente un $46,6 \%$ $(1-0,534=0,466)$. Sigue en importancia el índice de capital de trabajo $\left(C T \_A T\right)$ que por cada cambio unitario, disminuye la chance de crisis en un $23,8 \%$. También resultó significativo el índice de rotación $\left(V_{-} A T\right)$ al que le corresponde un odd ratio de 0,952 , lo que implica que la chance de ingresar a un estado de crisis disminuye en un $4,8 \%$ por cada cambio unitario en el índice. Por último, la estimación del índice de endeudamiento indica que por cada cambio unitario en el ratio, la chance de ingresar a un estado de crisis aumenta en un $1,7 \%$.

Con respecto al sector al que pertenecen las empresas existen diferencias si la empresa pertenece al sector industrial con respecto al sector de actividades primarias y de generación de energía, agua y gas, que fue utilizada como referencia. De tal forma si la empresa es del sector industrial tiene una altísima chance de estar en crisis respecto a la categoría de referencia, lo que puede ser explicado, en

\footnotetext{
${ }^{4}$ Odd ratio representa un riesgo, mediante el cociente entre el número de veces que ocurre el suceso (empresa en crisis) frente a cuántas veces no ocurre (empresa sana).
} 
parte por las empresas mineras (sector de referencia) que reciben significativos incentivos fiscales y constituyen el motor de las exportaciones chilenas.

En función de las predicciones realizadas por el modelo, se evalúa el mismo, a fin de determinar si las empresas son correctamente clasificadas en el estado correspondiente (Tabla 6).

Para ello, con procedimientos de post estimación, se determinó el punto de corte con métodos no paramétricos (Curvas ROC5) y se calcularon las tasas de clasificación correcta y las tasas de error.

El 96, 52\% de las empresas fue correctamente clasificado, mientras que el $3,45 \%$ fue clasificado incorrectamente como empresa sana a una empresa que se encuentra en crisis financiera (Error tipo I) y un 3,60\% el error de clasificar como empresa en crisis una empresa que se encuentra sana. El error tipo I resulta ser menor que el obtenido para las empresas argentinas (Tabla9).

Tabla 7: Porcentajes de clasificación - Chile.

\begin{tabular}{c|c}
\hline \hline Porcentajes & $\begin{array}{c}\text { Modelo con un efecto } \\
\text { Variables independientes: } \\
\text { ratios y tamaño }\end{array}$ \\
\hline Error tipo I & 3,45 \\
Error tipo II & 3,60 \\
$\%$ de clasificación correcta & 96,52 \\
\hline \hline
\end{tabular}

\subsection{Comparación con empresas de Argentina}

Los resultados obtenidos de la aplicación de estos métodos (Caro (2013))[7] se presentan en la Tabla 8 . Cabe aclarar que no se consideraron los sectores debido a la poca cantidad de empresas según el tipo de actividad.

$$
\begin{array}{r}
P\left(Y i=1 / \mathbf{X}_{i j}\right)=\beta_{0}+\left(\beta_{1}+b_{1 j}\right) G E_{-} A T_{i j}+\beta_{2} F F_{-} A T_{i j}+\beta_{3} E_{-} A T_{i j}+ \\
\beta_{4} V_{-} A T_{i j}+\beta_{5} D_{-} P N_{i j}+\beta_{6} C T_{-} A T_{i j}+\beta_{7} L N A T_{i j}
\end{array}
$$

con $\mathbf{X}_{i j}$ como vector de covariables.

Comparando este modelo con el modelo aplicado en las empresas chilenas, la primera diferencia que se obtiene es que el índice de rentabilidad ( $\left.G E \_A T\right)$

${ }^{5}$ Curvas ROC (Receiver Operating Characteristic): es una representación gráfica de la sensibilidad frente a (1-especificidad) para un sistema clasificador binario según se varía el umbral de discriminación. Permite seleccionar los modelos posiblemente óptimos y descartar modelos subóptimos independientemente de (y antes de especificar) el coste de la distribución de las dos clases sobre las que se decide. 
Tabla 8: Estimaciones de los parámetros-Argentina (Fuente: Caro (2013) [7]).

\begin{tabular}{|c|c|c|c|c|}
\hline $\begin{array}{l}\text { Efectos } \\
\text { Fijos }\end{array}$ & Coeficiente & $\begin{array}{c}\text { Error } \\
\text { Estándar }\end{array}$ & $\mathrm{p}$-value & $\begin{array}{c}\text { Odd Ratios variables } \\
\text { significativas }\end{array}$ \\
\hline Constante & 30,965 & 7,951 & 0,0003 & \\
\hline GE_AT & $-0,1473$ & 0,08 & 0,0739 & 0,86 \\
\hline FF_AT & $-0,0644$ & 0,0418 & 0.1306 & \\
\hline E_AT & 0,0467 & 0,2445 & 0.8494 & \\
\hline V_AT & $-0,0744$ & 0,0204 & 0.0007 & 0,93 \\
\hline D_Pn & 0,0157 & 0,0043 & 0.0007 & 1,02 \\
\hline CT_AT & 0,0234 & 0,0144 & 0.1069 & \\
\hline LNAT & $-1,551$ & 0,388 & $\mathbf{0 , 0 0 0 3}$ & 0,21 \\
\hline \multicolumn{3}{|c|}{ Efecto Aleatorio } & \multicolumn{2}{|c|}{$\begin{array}{l}\text { LR test vs logistic regression } \\
\text { Pr (chibar2) }\end{array}$} \\
\hline \multicolumn{3}{|l|}{ GE_AT } & \multicolumn{2}{|l|}{$\mathbf{0 , 0 0 0}$} \\
\hline
\end{tabular}

resulta significativo como efecto aleatorio en las empresas argentinas, mientras que en las chilenas lo es el índice de capital de trabajo.

Respecto a los efectos fijos se observa que los índices de rentabilidad $\left(G E \_A T\right)$, rotación $\left(V \_A T\right)$, endeudamiento $\left(D \_P N\right)$ y el tamaño de la empresa $(L N A T)$ fueron los que resultaron significativos para la estimación de las empresas en crisis. Puede concluirse que el índice de capital de trabajo $\left(C T_{-} A T\right)$ como medida del capital de corto plazo es una medida significativa para la predicción de crisis en las empresas chilenas, no siendo así en las empresas argentinas.

Es importante observar que los indicadores significativos en las empresas chilenas poseen mayor poder discriminatorio que las empresas argentinas, lo que se observa en el valor de los odd ratios. Siguiendo procedimientos de post estimación, la tasa de error tipo I (Tabla 9) es más elevada en las empresas argentinas que en las chilenas.

Tabla 9: Porcentajes de clasificación - Argentina.

\begin{tabular}{l|c}
\hline \hline \multicolumn{1}{c|}{ Porcentajes } & $\begin{array}{c}\text { Modelo con un efecto aleatorio } \\
\text { Variables independientes: } \\
\text { ratios y tamaño }\end{array}$ \\
\hline Error tipo I & 17,31 \\
Error tipo II & 0,54 \\
$\%$ de clasificación correcta & 95,80 \\
\hline \hline
\end{tabular}

Se concluye, entonces, que un modelo que predice adecuadamente crisis financiera en un mercado, no necesariamente lo hace en otro mercado. A su vez, 
hay ratios que ayudan a explicar el comportamiento de las empresas, aunque no posean el mismo poder discriminatorio, en cada uno de los países.

\subsection{Modelo con ratios en empresas chilenas}

Considerando como covariables, únicamente, los ratios contables, sin otras variables de contexto, el modelo aplicado en empresas chilenas es:

$$
\begin{array}{r}
P\left(Y_{i}=1 / \mathbf{X}_{i j}\right)=\beta_{0}+\left(\beta_{1}+b_{1 j}\right) \\
C T_{-} A T_{i j}+\beta_{2} G E_{-} A T_{i j}+\beta_{3} F F_{-} A T_{i j} \\
+\beta_{4} E_{-} A T_{i j}+\beta_{5} V_{-} A T_{i j}+\beta_{6} D \_P N_{i j}
\end{array}
$$

con $\mathbf{X}_{i j}$ como vector de covariables.

En este modelo el índice de capital de trabajo $\left(C T_{-} A T\right)$ es el efecto aleatorio (Tabla 10). Los mismos indicadores que resultaron significativos en el primer modelo lo son en éste y su poder predictivo es muy similar, ya que el índice de Rentabilidad ( $\left.G E \_A T\right)$, cuyo odd ratio de 0,576 , muestra que un incremento unitario en dicho índice disminuirá esa chance en aproximadamente un $42,4 \%$. Sigue en importancia el índice de capital de trabajo $\left(C T_{-} A T\right)$ que por cada cambio unitario, disminuye la chance de crisis en un $25,2 \%$. También resultó significativo el índice de rotación $\left(V \_A T\right)$ a un nivel de significación del 10\%, al que le corresponde un odd ratio de 0,979 , lo que implica que la chance de ingresar a un estado de crisis disminuye en un $2,1 \%$. Por último la estimación del índice de endeudamiento indica que por cada cambio unitario en el ratio, la chance de ingresar a un estado de crisis aumenta en un $0,7 \%$.

En función de las predicciones realizadas por el modelo, se evalúa el mismo, a fin de determinar si las empresas son correctamente clasificadas en el estado correspondiente (Tabla 11). En ambos modelos, las tasas de error son levemente superiores que las obtenidas en el modelo de la sección 4.1.

El modelo que considera sólo ratios como variables independientes posee los mismos ratios significativos que aquel primer modelo que incluye además tamaño y sector.

\section{Conclusiones}

La necesidad de evaluar el riesgo financiero de las empresas ha ido evolucionando con el tiempo. A partir de la segunda mitad del siglo pasado, la aparición de trabajos de predicción de crisis basados en la información contable han demostrado que ésta es de utilidad para anticiparse a situaciones de fracaso empresarial (Mínguez Conde, 2005) [12] que, sin duda es uno de los aspectos más preocupantes, no sólo para el empresario, sino para la sociedad en general, poniendo 
Tabla 10: Estimación de los parámetros del modelo logístico mixto (solo ratios contables) - Chile.

\begin{tabular}{|c|c|c|c|c|}
\hline Covariables & Coeficiente & $\begin{array}{c}\text { Error } \\
\text { Estándar }\end{array}$ & $P>z$ & $\begin{array}{l}\text { Odd ratios de } \\
\text { variables } \\
\text { significativas }\end{array}$ \\
\hline GE_AT & $-0,5512$ & 0,1097 & 0,0001 & 0,576 \\
\hline FF_AT & $-0,0421$ & 0,0397 & 0,2910 & \\
\hline E_AT & 0,3729 & 0,2529 & 0,1432 & \\
\hline V_AT & $-0,0216$ & 0,0115 & 0,0639 & 0,979 \\
\hline D_Pn & 0,0066 & 0,0030 & $\mathbf{0 , 0 3 1 3}$ & 1,007 \\
\hline CT_AT & $-0,2908$ & 0,0801 & 0,0005 & 0,748 \\
\hline Constante & $-1,2764$ & 0,3807 & 0,0011 & \\
\hline $\begin{array}{l}\text { Efecto } \\
\text { Aleatorio }\end{array}$ & Varianza & $\begin{array}{c}\text { Error } \\
\text { Estándar }\end{array}$ & \multicolumn{2}{|c|}{$\begin{array}{l}\text { LR test vs logistic regression } \\
\text { Pr (chibar2) }\end{array}$} \\
\hline CT_AT & 0,2237 & 0,0839 & \multicolumn{2}{|r|}{0,000} \\
\hline
\end{tabular}

Tabla 11: Porcentajes de clasificación - Chile.

\begin{tabular}{l|c}
\hline \hline \multicolumn{1}{c|}{ Porcentajes } & $\begin{array}{c}\text { Modelo con un efecto aleatorio } \\
\text { Variables independientes: ratios }\end{array}$ \\
\hline Error tipo I & 5,17 \\
Error tipo II & 4,50 \\
$\%$ de clasificación correcta & 94,94 \\
\hline \hline
\end{tabular}

de relieve los factores explicativos de tales situaciones. El acceso a la información contable y el uso de herramientas estadísticas cada vez más potentes han contribuido al análisis de esta problemática.

La crisis financiera de las empresas ha sido y es un tema de preocupación en el mundo. De hecho, en Argentina los estudios preliminares que se realizaron (Sandín y Porporato, 2007 [16] y Díaz et al. 2001[10]) desafiaron los modelos internacionales que han sido aplicados en diferentes países (Altman, 1993) [4], ya que se lograron mejores resultados en las predicciones cuando se utilizaron modelos propios aplicados a la economía argentina. Luego se aplicaron modelos mixtos con mejor performance que los modelos para corte transversal (Caro et al. 2013 [10] y Caro, 2013 [7]).

En síntesis, se concluye que, debido a que la estructura de los datos introduce dependencia en las respuestas múltiples dentro de cada unidad, es necesaria la aplicación de un modelo mixto. De esta manera se responde a la pregunta de investigación formulada, ya que son los ratios contables, los indicadores válidos para predecir la crisis financiera. 
Ambos mercados permiten caracterizar las empresas según la existencia o no de crisis financiera y analizar los ratios contables que inciden en su probabilidad.

En las empresas chilenas, el ratio del capital de trabajo explica la mayor proporción de la heterogeneidad inducida por la correlación que presentan los datos, lo que justifica su inclusión como coeficiente aleatorio.

En el modelo que incluye tanto ratios financieros como características de la empresa, los indicadores con mayor capacidad predictiva de la crisis financiera son el tamaño, los índices de rentabilidad, rotación, capital de trabajo y endeudamiento.

Comparativamente con el modelo aplicado a las empresas argentinas, algunos índices son coincidentes, a excepción de los ratios de capital de trabajo y el de flujo de fondos, ya que el primero resulta significativo en las empresas chilenas y no en las argentinas, mientras que el segundo resulta significativo en las argentinas y no en las chilenas.

Respecto al modelo construido con las empresas chilenas que solo incluye a los ratios financieros, los indicadores significativos fueron los mismos, por lo que se concluye que éstos poseen poder discriminatorio y su comportamiento muestra que son indicadores para la predicción de crisis.

Por lo expuesto, podemos responder el interrogante planteado, ya que los ratios financieros son indicadores válidos para predecir la crisis financiera.

\section{Referencias}

[1] Altman, E. (1968) "Financial ratios, discriminant analysis and the prediction of corporate bankruptcy", Journal of Finance 23(4): 589-609.

[2] Altman, E.; Haldeman, R.; Narayanan, P. (1977) "ZETA analysis: a new model to identify bankruptcy risk of corporations", Journal of Banking and Finance 1(1): 29-54.

[3] Altman, E.I,; Baidya, T.K.N.; Ribeiro Dias, L.M, (1979) "Assesing potential financial problems for firms in Brazil", Journal of International Business Studies 10: 9-24.

[4] Altman, E.; Hotchkiss, E. (2005) Corporate Financial Distress and Bankruptcy: Predict and Avoid Bankruptcy, Analyze and Invest in Distressed Debt, 3rd Ed. Wiley Finance, New York.

[5] Beaver, W. (1966) "Financial ratios as predictors of failures", Journal of Accounting Research, Supplement 4, Empirical Research in Accounting Selected Studies 1966: 71-111. 
[6] Beaver, W. (1968) "Alternative accounting measures as predictors of failure", The Accounting Review 43(1): 113-122.

[7] Caro, N.P. (2013) Evaluación de Riesgo de Crisis Financieras en Empresas Argentinas en los Periodos 1993-2000 y 2003-2010. Tesis Doctoral, Facultad de Ciencias Económicas, Universidad Nacional de Córdoba.

[8] Caro, N.P.; Díaz, M.; Porporato, M. (2013) "Predicción de quiebras empresariales en economías emergentes: uso de un modelo logístico mixto", Revista de Métodos Cuantitativos para la Economía y la Empresa 16: 200215.

[9] Caro, N.P. (2014) "Modelos de predicción de crisis financiera en empresas: una revisión de la literatura”, Revista Internacional Legis de Contabilidad y Auditoría 58: 135-183.

[10] Díaz, M.; Ferrero, F.; Stimolo, M.; Caro, N. (2001) "Performance del análisis discriminante regularizado y la regresión logística en la predicción de crisis financiera", Revista de la Sociedad Argentina de Estadística 5 (1-2), $33-45$.

[11] Jones, S.; Hensher, D.A. (2004) "Predicting firm financial distress: a mixed logit model", The Accountig Review 79(4): 1011-1039.

[12] Mínguez Conde, J.L. (2005) La Información Contable en la Empresa Constructora: Factores Identificativos del Fracaso Empresarial. Tesis doctoral, Universidad de Valladolid.

[13] Mongrut Montalván, S.; Alberti Delgado, F.I.; Fuenzalida O’Shee, D.; Akamine Yamashiro, M. (2011) "Determinantes de la insolvencia empresarial en el Perú", Academia, Revista Latinoamericana de Administración 47: 126-139; in: http://www.redalyc.org/src/ inicio/ArtPdfRed.jsp?iCve=71618917009 ISSN 1012-8255, consultada 20-Feb-2014.

[14] Pascale, R.(1988) "A multivariate model to predict firm financial problems the case of Uruguay", Studies in Banking and Finance 7: 171-182.

[15] Romani Chocce, G.; Aroca González, P.; Aguirre Aguirre, N.; Leiton Vega, P.; Muñoz Carrazana, J. (2002) "Modelos de clasificación y predicción de quiebra de empresas: una aplicación a empresas chilenas", Forum Empresarial 7(1): 33-50. in: http://www.redalyc.org/pdf/631/ 63170102 .pdf, consultada 12-Feb-2014. 
[16] Sandin, A.R.; Porporato, M. (2007) "Corporate bankruptcy prediction models applied to emerging economies. Evidence from Argentina in the years 1991-1998", International Jorunal of Commerce and Management 17(4): 295-311.

[17] Swanson, E.; Tybout, J. (1988) "Industrial bankruptcy determinants in Argentina”, Journal of Banking and Finance 7: 1-25.

[18] Zurita, F. (2008) "La predicción de la insolvencia de empresas chilenas", Economía Chilena 11(1): 93-116. 